\title{
DETECTION, CAUSES AND WAYS OF PREVENTION OF CAVITATION IN PUMPS
}

\author{
Harshal Patil \\ Mechanical Engineering Department \\ Institute of Technology, Nirma University, Ahmedabad, India
}

\begin{abstract}
Pumps and turbines are an integral part of the hydro machine family and play a vital role in many large industries. These machines are required to operate efficiently for the maximum output, and thus they must be free from any damages or problems. Cavitation is a phenomenon occurring in hydro machines (more specifically, pumps and turbines) which is related to the change and fluctuation in the pressure of fluids, the same phenomenon further leads to cavitation erosion. It turns out to be disadvantageous for the machines and deteriorates their performance and efficiency. This paper consists of a detailed study about the phenomenon, including how can we detect the damage and root of the problem. Further, their causes are discussed with the ways of prevention. This will help in taking necessary measures from the beginning so that the problems with the machines can be avoided.
\end{abstract}

Keywords: Cavitation, Pumps, Cavitation erosion, NPSH

\section{INTRODUCTION}

Pumps and turbines are very widely used hydro machines. As the word "hydro" already suggests, these machines work in and around fluids and are always surrounded by them. The main rotating part in these hydro machines are impellers which consist of blades for functionality. Impeller blades are the ones that are in maximum contact with the operating fluids and hence, are at high risk of possible damage. Cavitation is the most common damaging phenomenon among all.

\section{I.1 Cavitation}

When the pumps and turbines are operation, the impeller blades are often the parts which get pitted during the functionality. They develop pores and roughness, degrading the metal surface. The pitting of the metal surface is known as "Cavitation". When the pressure at any point in liquid drops below vapor pressure, the phenomenon taking place in the pump is called cavitation. It is the process of vaporization, bubble generation and bubble implosion which occurs in the liquid in operation of pump as a result of a decrease and subsequent increase in local pressure. We look further to understand it more clearly:

Vapor pressure is defined as the pressure exerted by vapor particles when the vapor is in equilibrium with the liquid or solid form. Boiling temperature of any fluid keeps fluctuating with temperature. As per Charles' Law, we know that pressure is directly proportional to temperature. Thus, with increase in vapor pressure temperature also increases and same way, temperature decreases with decrease in vapor pressure. The inlet of pumps produce suction on the fluid which creates vacuum. Pressure at that particular point drops down due to vacuum, thus decreasing the temperature. This decrease in temperature results in the formation of air bubbles which collide with the metal surface of impeller blades, and this is how cavitation takes place. These bubbles damage and erode the blades.

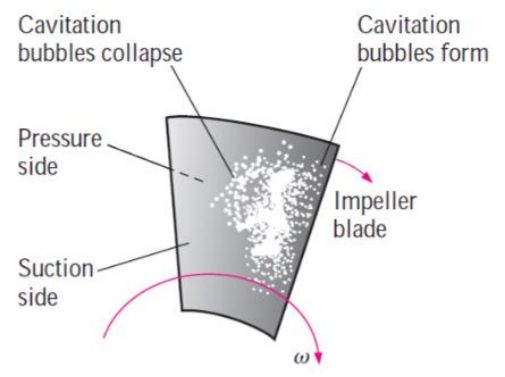

Fig 1: Cavitation bubbles forming and collapsing on the suction side of an impeller blade. [1]

\section{I.2 Erosion due to cavitation}

Also referred to as pitting erosion, cavitation erosion occurs mainly on the low-pressure side of the impeller blades. The excess cavitation results in this erosion and it results in degradation of impeller blades' surface. Pitting depth on the surface sometimes exceeds $40 \mathrm{~mm}$ which creates barrier on the performance of the machines. As large as $90 \%$ hydro machines suffer damage due to cavitation to at least some extent.

The average metal loss due to erosion is around $5 \mathrm{gs} / \mathrm{m} 2 / 10,000$ hours. A turbine runner used over a period of years may suffer metal losses up to $200 \mathrm{~kg}$. [2]

Mainly three types of devices are used to study the cavitation erosion: [2]

i) Rotating disk

ii) Hydrodynamic tunnel

iii) Vibratory device producing cavitation

\section{DETECTION}

To resolve a problem, we must first detect it. In this section, we will discuss the ways to detect cavitation in a system. There are some symptoms which give a hint about 


\section{International Journal of Engineering Applied Sciences and Technology, 2021 \\ Vol. 6, Issue 3, ISSN No. 2455-2143, Pages 88-90 \\ Published Online July 2021 in IJEAST (http://www.ijeast.com)}

the present cavitation in a pump. The signs to look for to detect cavitation are:

\subsection{Excessive vibration:}

When cavitation takes place, the pump experiences more vibrations than usual. The unstable flow due to the explosion of bubbles causes this vibration.

\subsection{Noise:}

The collisions of the air bubbles make loud, unusual and crackling noises; this is an obvious sign to look for.

\subsection{Increased power consumption:}

The machine may consume more power than usual. This may occur due to cavitation.

\subsection{Decrease in performance and efficiency:}

This includes the phenomenon like reduction in discharge pressure than normally observed by the operator.

\subsection{Debris in the discharged liquid:}

In extreme case of cavitation, this phenomenon could be observed.

\section{CAUSES}

There are multiple causes due to which cavitation may occur. But the root cause of all remains the same, that is: pressure of liquid at a point decreasing below the vapor pressure. Various other causes of the problem have been discussed below:

\subsection{Pump operating at a higher speed:}

This is an important cause of cavitation, this results in the fluctuation of temperature and rapid movements of bubbles thus striking the blade surfaces.

\subsection{Too small diameter of suction pipe:}

This results in pressure fluctuation, thus causing cavitation.

\subsection{Smaller Net Positive Suction Head (NPSH) than} required:

Cavitation can also occur when the actual NPSH of the pump is smaller than that of the required NPSH.

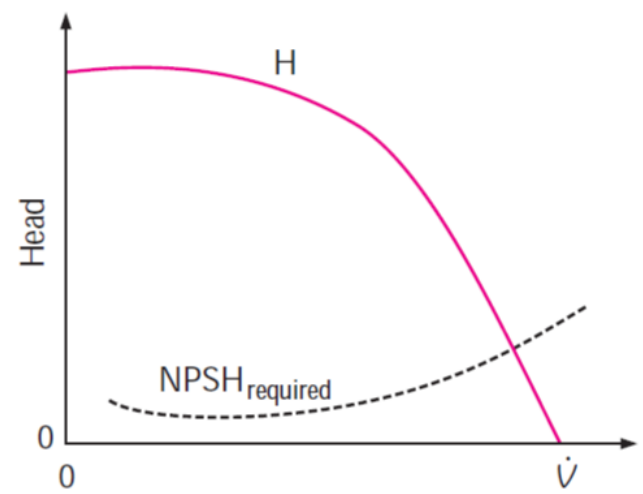

Fig 2: Typical pump performance curve (NPSH vs volume flow rate). [1]

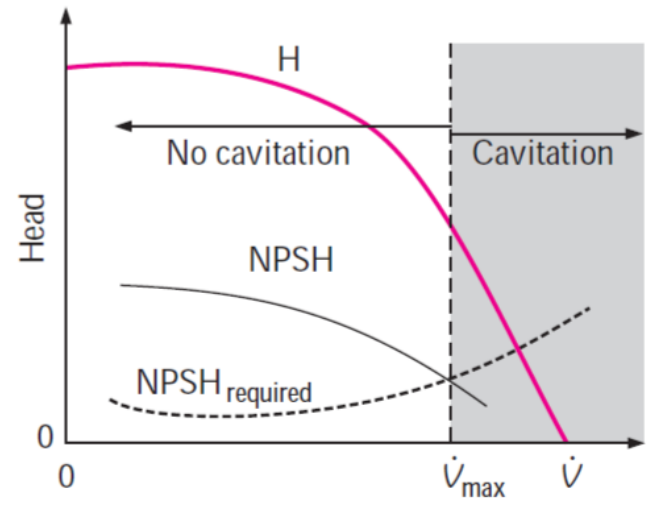

Fig 3: Volume flow rate where actual and required NPSH intersect, represents maximum flow rate delivered by pump without cavitation. [1]
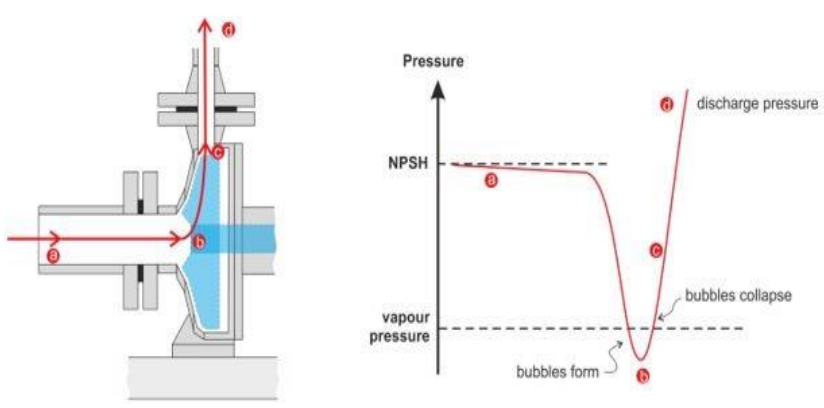

Fig 4: Different points in the pump with their pressure comparison with vapor pressure. [3]

\section{WAYS OF PREVENTION OF CAVITATION}

It is utmost important to prevent cavitation to the best extent we can. Hence, in this section, the possible ways to prevent cavitation are discussed. Some of them include:

\subsection{To reduce friction losses:}

This can be executed by using pumps of large diameters.

\subsection{Using negative suction pumps:}

Negative suction pumps are used at various places where there are chances of cavitation. This prevents the interference of vapor and fluid. Foot valves (NRV) are used at suction to avoid the need of priming frequently.

\subsection{To control the pressure in the flow:}

To avoid cavitation, the pressure in the flow should be controlled and must not be allowed to drop below the vapor pressure.

\subsection{To ensure actual NPSH is greater than required NPSH:}

To prevent the risk of cavitation, it is advised to maintain a margin of at least $0.5 \mathrm{~m}$ between the two. [3] 


\section{International Journal of Engineering Applied Sciences and Technology, 2021 \\ Vol. 6, Issue 3, ISSN No. 2455-2143, Pages 88-90 \\ Published Online July 2021 in IJEAST (http://www.ijeast.com)}

4.5 To reduce the temperature at intake:

Reducing temperature at the intake prevents the fluctuations in the temperature and pressure.

\subsection{Decreasing pump speed:}

The speed of the pump can be decreased to prevent cavitation.

4.7 Regular maintenance and use of anti-cavitation material:

This can ensure the machine to operate smoothly. Material such as stainless steel can be considered.

\section{CONCLUSION}

It is tough to completely tackle cavitation. As some experts suggest, in spite of enhancing the design and material of the pump, cavitation cannot be prevented totally. But we can still take the best steps possible to minimize it as much as we can. Cavitation can surely be reduced to economically accepted level without affecting the performance to machines to a much great extent.

\section{REFERENCES}

[1] C. Yunus and C. John, Fluid Mechanics: Fundamentals and Applications, Mc Graw Hill Education, 2004.

[2] R. Akash and J. Amit, "A Review Paper on Hydrodynamic Cavitation," International Journal of Engineering Science and Computing (IJESC), vol. 7, no. 4, 2017.

[3] "Useful information on pump cavitation," michaelsmith-engineers.co.uk. 